DW. ALS in Rochester, Minnesota, 1925-1977. Neurology 1980;30:463-70.

4 Yoshida S, Mulder DW, Kurland LT, Chu C-P, Okazaki H. Follow-up study on amyotrophic lateral sclerosis in Rochester, Minnesota, 1925 through 1984. Neuroepidemiology, (in press).

5 Kurland LT, Choi NW, Sayre GP. Implications of incidence and geographic patterns on the classification of amyotrophic lateral sclerosis. In: Norris FH Jr, Kurland LT, eds. Motor Neuron Diseases. New York: Grune \& Stratton, 1969:28-50.

\section{Li et al reply}

We were interested to see Drs Kurland and Mulder's comments on age specific incidence rates in motor neuron disease, and note that our findings are in agreement with theirs. Clearly, this implies a common causative factor operating in these widely separated environments and it would be interesting to compare cohort data for these different populations. We agree with Kurland and Mulder that the diagnosis of motor neuron disease is probably often overlooked in elderly patients, thus resulting in an under-estimate of incidence rates in older populations. This is a common limitation of epidemiological data taken from retrospective surveys of case-notes, but in our work we have tried to exclude this factor as far as possible by utilising data from two separate Health Authorities, both with relatively well-organised neurological services. Similar trends were found in the two sets of data.

\section{Cryptococcal meningitis and cerebral toxo- plasmosis in a patient with acquired immunodeficiency syndrome.}

Sir: We were very interested in the short report of Bahls and Sumi ${ }^{1}$ about the documented simultaneous infection of the central nervous system with Toxoplasma gondii and Cryptococcus neoformans in an AIDS patient. We have also observed such an association on the same underlying disease.

A 26 year old homosexual man was admitted with fever, cough and meningitis. Transbronchial biopsy, blood and CSF cultures showed Cryptococcus neoformans. CD4/CD8 lymphocytes ratio was under $0 \cdot 2$. Serum sample was LAV/HTLV III antibody positive by two different techniques (ELISA, Western blott). Despite amphotericin B and 5-fluorocytosine in combination, seizures and confusion with right hemiparesis appeared. The cranial computed tomographic scan revealed three ring-enhancing mass lesions. Echoguided neurosurgical puncture of the left parietal mass lesion allowed brain biopsy which showed Cryptococcus neoformans (PAS stain). The patient died 4 weeks later. Toxoplasmosis serological and CSF tests were non-diagnostic. Brain culture from mice after intraperitoneal inoculation were positive for Toxoplasma gondii.

This new case emphasises the possibility of an infectious agent hiding another. Brain biopsy is indicated in such patients because of the lack of correlation between clinical presentation, CT scan appearance of mass lesions in the central nervous system, isolation of an infectious agent anywhere and the specific diagnosis of mass lesions. ${ }^{2}$ In this case, however, despite a brain biopsy in an affected area, it was not very useful, because routine haematoxylin and zosin stain was negative for $\boldsymbol{T}$ gondii, and culture from laboratory animal was necessarily slow. The immunohistological staining with peroxydase anti-peroxydase stain methodfast and specific diagnosis procedure for $T$ gondii $^{3}$ must be recommended, particularly in immunodeficient patients needing a rapid and specific diagnosis, critical to the directing of appropriate and urgent therapy for a potentially curable condition.

F LUCHT

$J$ JAUBERT

J HUPPERT

R TRAN MAHN SUNG

JC LEPETIT

H ROUSSET

Hospital de Belleune, .Chru de St Etienne, Blvd Pasteur, 42023 St Etienne Cedex, France.

\section{References}

${ }^{1}$ Bahls F, Sumi SM. Cryptococcal meningitis and cerebral toxoplasmosis in a patient with acquired immunodeficiency syndrome. $J$ Neurol Neurosurg Psychiatry 1986;49: 328-30.

${ }^{2}$ Levy RM, Pons VG, Rosenblum ML. Intracerebral mass lesions in the acquired immunodeficiency syndrome. $N$ Engl J Med 1983;309:1454-5.

${ }^{3}$ Conley FK, Jenkins KA, Remington JS. Toxoplasma gondii infection of the central nervous system: use of the peroxydase antiperoxydase method to demonstrate Toxoplasma in formalin-fixed, paraffin embedded tissue specimen. Hum Pathol 1981:12:690-8
Cryptococcal meningitis and cerebral toxo- $\bar{Z}$ plasmosis in AIDS: another case report.

Sir: We read with interest the Short report of Bahls and Sumi ${ }^{1}$ on the association of cryp- $\frac{}{0}$ tococcal meningitis and cerebral toxoplasmosis in a patient with Acquired Immune Deficiency Syndrome (AIDS) and we would like to document a further case.

A 34 year old homosexual male was admitted with a 4 months history of slight fever, cough with occasional haemoptysis $\overline{\bar{\omega}}$ and weight loss. Three months later he developed a headache. One day before admission the patient became less alert. Neurological examination showed a comatose patient with nuchal rigidity and left facial paralysis. The diagnosis of AIDS and cryptococcal meningitis was made by clinical and laboratory evaluation and was treated with amphotericine $B$ and 5-fluorocytosine. After $2=$ months he developed progressive hemi- $\omega$ paresis and CT scan showed multiple, ringenhancing, low-density lesions. Slightly increased serologic titres against Toxoplasma gondii were detected and therapy with pyrimetamine and sulfonamides was consequently started. In the next week the patient quickly improved, the CT lesion disappeared and he soon became asympor tomatic. Unfortunately, after one and a hafg months, therapy was discontinued because severe pancytopenia had developed, and he progressively experienced again right limb weakness and aphasia. Later, he died pneumonitis from Pneumocystis carin Pathological findings in the central nervou system revealed cryptococcal meningoencephalitis, multiple necrotising abscesses for Toxoplasma gondii and medulla and spinal cord infection by cytomegalovirus.

In our opinion, taking into account that cryptococcus and toxoplasma are both common causes of infection in patients with AIDS, ${ }^{2-5}$ their association may frequently occur. Consequently, we suggest the immediate onset of therapy with pyrimethamine and sulfadiazines if the diagnosis of toxoplasmosis is suspected ${ }^{5}$ in patients with cryptococcal meningitis and AIDS. Brain biopsy should be reserved for cases with poor response to treatment because its use is limited by its potential morbility and the presence of false-negative results.

I BONAVENTURA IBARS

J MATIAS-GUIU

C CERVERA

A CODINA PUIGGROS

Neurology Service of the

Hospital del Valle de Hebrón,

Universidad Autónoma de Barcelona,

Spain 
References

I Bahls F, Sumi SM. Cryptococcal meningitis and cerebral toxoplasmosis in a patient with acquired immune deficiency syndrome. $J$ Neurol Neurosurg Psychiatry 1986;49: 328-30.

2 Snider W, Simpson D, Nielsen S, et al. Neurological complications of Acquired Immune Deficiency Syndrome: analysis of 50 patients. Ann Neurol 1983;14:403-18.

3 Koppel B, Wormser G, Tuchman AJ, et al. Central nervous system involvement in patients with acquired immune deficiency syndrome. Acta Neurol Scand 1985;71: 337-53.

4 Levy RM, Bredesen DE, Rosenblum MI. Neurological manifestations of the acquired immuno deficiency syndrome: experience at UCSF and review of the literature. $J \mathrm{Neu}$ rosurg 1985;62:475-95.

5 Navia BA, Petito CK, Gold JW, et al. Cerebral toxoplasmosis complicating the acquired immune deficiency syndrome: clinical and neuropathological findings in 27 patients. Ann Neurol 1986;19:224-38.

Paroxysmal myoclonic dystonia with vocalisations

Sir: Feinberg et al ${ }^{1}$ described four patients whom they distinguished from the large majority of patients with Gilles de la Tourette syndrome on the basis of four "atypical" characteristics: (1) paroxysmal bursts of regular, repetitive, rhythmic, stereotypic, coordinated, simultaneous and bilateral myoclonus and vocalisations often with tonic symptoms; (2) presence of hyperactivity and attentional and learning disorders; (3) interference of symptoms with voluntary functioning; and (4) ineffectiveness of haloperidol. I have examined Case 1 and have considered him not to have the lightning-like jerks of myoclonus. Rather, he has virtually all the features of Gilles de la Tourette syndrome. I presented videotapes of his paroxysmal bursts of abnormal movements at the Unusual Movement Disorder Seminar held 29 May 1986, at the meeting of the American Academy of Neurology. The audience of 160 neurologists was in complete agreement that this young man suffered from the tic syndrome, commonly known as Gilles de la Tourette syndrome.

Let me address the four features of the disorder that the authors considered atypical. The first are the "paroxysmal bursts of regular, repetitive, rhythmic, stereotypic, coordinated, simultaneous and bilateral abnormal myoclonus (sic) and vocalisations often with tonic symptoms." Paroxysmal bursts of stereotypic and coordinated move- ments are the hallmark of tics, ${ }^{2}$ and, in fact, are not encountered in other movement disorders. Paroxysmal dyskinesias of dystonia and chorea are well recognised, ${ }^{3}$ and paroxysmal tremor has been reported, ${ }^{4}$ but none of these are coordinated sequences of complex movements that are so typical of tics. Vocalisations, also, are a classical feature of the Gilles de la Tourette syndrome, and are only encountered elsewhere in Meige syndrome and as a feature of akathisia. The vocalisations of Case 1 included coprolalia, which is almost diagnostic for Gilles de la Tourette syndrome. Bursts of repetitive, rhythmic, bilateral movements are not commonly seen as part of the motor tic spectrum, but I see no reason why this phenomenology cannot be included within the realm of motor tics. Indeed, a minority of patients seen by me with otherwise typical features of tics have this feature. Tonic symptoms have long been recognised as a feature of tics, ${ }^{256}$ and today are commonly referred to as dystonic tics.

It is not clear why Feinberg et al listed "presence of hyperactivity and attentional and learning disorders" as atypical for patients with tics. Several investigators report that attention deficit disorder occurs in approximately $50 \%$ of patients with Gilles de la Tourette syndrome. ${ }^{7-9}$

Interference of voluntary functioning by symptoms does occur in Case 1. When he has a burst of the repetitive, rhythmical flexion movements of his arms, he stops speaking, other than occasional vocalisations. There is no loss of contact with the environment; rather, it appears as if his mind is actively and compulsively engaged in other activity, which is what he and other patients with tics who have this symptom inform me. Like bursts of repetitive movements, these simultaneously mindoccupying states should be considered within the spectrum of tics.

The final point raised by Feinberg et al is the ineffectiveness of haloperidol to suppress these paroxysmal motor bursts. But responsivity to medication is not an acceptable criterion for the diagnosis of tics! As an aside, and for a point of information, based on a telephone conversation I had with the patient on 8 May 1986, he considers himself $80 \%$ improved on fluphenazine and clonidine. Thus, the former drug, which blocks dopamine, similar to haloperidol, has benefitted the patient.

I would like to propose that authors who wish to describe new or variants of movement disorders should also submit a videotape showing the abnormal movements. The demonstration of the videotape can most easily be accomplished in the newly founded journal Movement Disorders which includes a video format in addition to the classical written format. Those interested in this new journal can contact its publisher, Raven Press. I have obtained written permission from Case 1 to publish his videotape, and I will append it as part of the review of tics by Jankovic and Fahn. ${ }^{6}$

STANLEY FAHN Neurological Institute, 710 West 168th Street New York, NY 10032.

USA

\section{References}

1 Feinberg TE, Shapiro AK, Shapiro E. Paroxysmal myoclonic dystonia with vocalisations: new entity or variant of preexisting syndromes? J Neurol Neurosurg Psychiatry 1986;49:52-7.

2 Fahn S. The clinical spectrum of motor tics. Adv Neurol 1982;35:341-4.

3 Lance JW. Familial paroxysmal dystonic choreoathetosis and its differentiation from related syndromes. Ann Neurol 1977;2: 285-93.

4 Fahn S. Paroxysmal tremor. Neurology 33 (Suppl 2) 1983:131.

5 Meige H, Feindel ECL. Tics and their treatment. Translated from the French by Wilson SAK. London: Appleton, 1907.

6 Jankovic J, Fahn S. The phenomenology of tics. Movement Disorders 1986;1:17-26.

7 Shapiro AK, Shapiro ES, Bruun RD, Sweet RD. Gilles de la Tourette Syndrome. New York: Raven Press, 1978.

8 Nee LE, Caine ED, Polinsky RJ, Eldridge R, Ebert MH. Gilles de la Tourette syndrome: Clinical and family study of $\mathbf{5 0}$ cases. Ann Neurol 1980;7:41-9.

9 Cohen DJ, Detlor J, Shaywitz BA, Leckman JF. Interaction of biological and psychological factors in the natural history of Tourette syndrome: a paradigm for childhood neuropsychiatric disorders. Adv Neurol 1982;35:31-40.

\section{Feinberg et al reply}

We are pleased to respond to Dr Fahn's comments on our paper. The fact that Case No 1 was presented at the Academy of Neurology meetings highlights the atypical nature of the symptoms. We also have presented Case No 1, in addition to the three other patients described in our report, including videotapes, to neurologists interested in movement disorders at the University of Michigan Medical School, Mount Sinai School of Medicine and the Basal Ganglia Club in New York City. None of over 150 physicians at any of these meetings opined that the symptoms represented Gilles 